\title{
A. Veves, J.M. Giurini, F.W. LoGerfo (eds) The diabetic foot (second edn). Humana Press, Totowa, New Jersey, 2006
}

\author{
743pp (ISBN 158829610 5). Hardcover \$185
}

\section{R. Landgraf}

Received: 31 October 2006 / Accepted: 14 December 2006 / Published online: 20 January 2007

(C) Springer-Verlag 2007

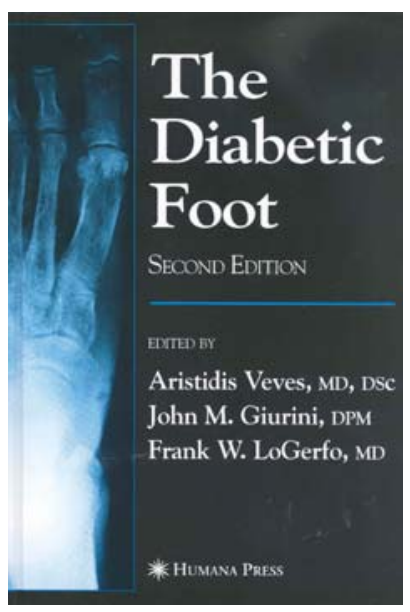

The extensive literature on the diabetic foot syndrome (DFS) that has appeared over the past decade reflects the importance of this serious complication and its socioeconomic burden.

This book was first published more than 4 years ago and is now in its second edition, and it includes chapters written by many well-known specialists. It provides comprehensive coverage of many important aspects of DFS, such as epidemiology, healthcare costs, wound-healing processes and skin regeneration, diabetic neuropathy, microangiopathy of the diabetic foot, diagnosis of macrovascular disease, and clinical and technical examinations (foot pressure measurements, imaging of the foot, etc.). Several

R. Landgraf $(\bowtie)$

Diabetes Centre, Medizinische Klinik Innenstadt,

University of Munich,

80336 Munich, Germany

e-mail: ruediger.landgraf@gmx.de chapters deal with state-of-the-art management of patients with DFS, including the wide range of options on wound care, surgery of the foot, soft tissue reconstruction, amputations and lower extremity arterial reconstruction (surgical as well as angioplasty). Psychosocial aspects, training, and education and preventive measures are also discussed. An extensive reference list follows each of the 25 chapters.

As with many textbooks, this book is excellent in parts, while other parts are not of the same high standard. The first chapter sets out to deal with the principles of care for the surgical patient with diabetes. In reality, however, the epidemiology, classification and pathophysiology, acute and chronic complications, and the management of type 1 and type 2 diabetes are discussed. Very few of the 30 pages of this chapter are concerned with the management of the patient at high risk and during surgery. This particular chapter is a little too broad for many clinicians, particularly for the specialist in foot problems.

Chapter 2 on epidemiology and healthcare costs for diabetic foot problems is important, since it contains reliable but not easily obtainable data from various healthcare settings.

Chapters 3 and 4 discuss wound healing. Both chapters could be more concise. They are difficult to assimilate, since they contain no tables or figures. Data from research on humans and animals are presented together, making it hard to identify information relevant to the clinician. Again, colour figures would have been helpful to the reader in the section dealing with regeneration of skin and peripheral nerves, as would a clearer separation of experimental and clinical data.

The chapter on diabetic neuropathy (Chapter 6), an important aspect of diabetic foot syndrome, is very comprehensive and displays much of the relevant data in 
tables. However, the discussion of the management of neuropathy is too short: an algorithm for treatment, more details on side effects, and the numbers needed to treat for the various options in the treatment of autonomic and peripheral sensory-motor polyneuropathy would have been useful additions.

Microvascular changes in the diabetic foot, which is a topic still under extensive debate, are discussed in detail. The diagnostic methods to evaluate microcirculation are listed, but there is no critical summary of the sensitivity and specificity of the methods.

The chapters on macrovascular disease (Chapters 8, 22 and 23) are very welcome and up-to-date, but the contents are not well organised. It would have been preferable to have included epidemiology, clinical features, diagnosis and treatment options in a single chapter. The chapter on foot pressure and on the methods for measuring foot pressure abnormalities are well-written comprehensive reviews, as is the chapter on the biomechanics of the diabetic foot.

The chapter on the clinical examination of the diabetic foot covers all relevant aspects well, but includes material that has already been discussed in other chapters (for example, vascular evaluation). Again, the contents of this chapter could have been better organised; it is also a pity that the figures are in not colour. The chapter on imaging of the diabetic foot provides a critical discussion on the indications and limitations of methods for the diagnosis of the multiple abnormalities of the diabetic foot. However, the importance and diagnostic sensitivity of current ultrasound methods (Doppler/Duplex) are not covered in detail. The section on angiography is too brief and there are no cross-references to the other chapters in which angiography is discussed.

Chapter 13, on the microbiology and treatment of foot infections, is inserted between the chapters on imaging and Charcot arthropathy, although the chapters on local and systematic treatment follow later. Chapters on wound preparation, local care of ulcers, surgical procedures, amputation and tissue reconstructive options cover all the important points, but would have been enhanced by colour figures. The book ends with two important chapters on psychosocial, educational and preventive aspects.

Overall, this textbook is worth reading and having readily available: it provides up-to-date information on many aspects of this important and increasingly common condition. However, for daily clinical practice and for quick reference, there is too much text, and too few tables, figures, and algorithms for diagnosis and treatment options. In addition, too few critical summaries are included. Because there are multiple authors, there is considerable overlap on several topics between different chapters. It is a shame that in a book published in 2006 there is almost no mention of the evidence grades for the different diagnostic and therapeutic options discussed. It is also remarkable that there is no chapter on quality management and the organisation of integrated care, one of the most neglected aspects of healthcare. 